\title{
Design of a Marx Generator for HEMP Filter Evaluation Taking Account of Parasitic Effect of Components
}

\author{
H. Jiang*, J. Zhou*, Y. Huang*, Y. Zeng*, J. Humphries*, Q. Xu*, C. Song*, L. Xing ${ }^{\#}$ and \\ J. Nalborczyk ${ }^{\dagger}$ \\ *Univeristy of Liverpool, UK, jiafeng.zhou@liverpool.ac.uk, yi.huang@liverpool.ac.uk \\ ${ }^{\#}$ Nanjing University of Aeronautics and Astronautics, China \\ ${ }^{\top}$ MPE Limited,UK, JNalborczyk@mpe.co.uk
}

\begin{abstract}
This paper introduces the design of a Marx Generator that can generate high attitude pulses up to $25 \mathrm{kV}$ and $2.5 \mathrm{kA}$. The pulses have a wave shape representative of that used to test High-altitude ElectroMagnetic (HEMP) filters against the pulse current injection (PCI) requirements. The rise time is $20 \mathrm{~ns}$ and the pulse width is 230 ns on a $10 \Omega$ resistor.

In the design, it was found that parasitic effect of circuit components could significantly affect the performance of the generator. This is mainly because the pulse width is very narrow and its frequency spectrum is very wideband. The high-frequency circuit models of the components used in the generator were obtained by calculation and optimization, and then further verified by experiment. The verified circuit models were then used to design the three-stage generator. The measured performance agrees very well with the simulated one using the proposed circuit models.
\end{abstract}

Keywords: HEMP, EMC, Parasitic, Pulse, Circuit Model, Marx Generator.

\section{Introduction}

High-altitude ElectroMagnetic Pulse (HEMP) filters help to safeguard equipment and systems against the potentially devastating effects of HEMP generated in the atmosphere [1]. Military computer and communications networks as well as civil and commercial infrastructure can be knocked out by HEMP if they are not adequately protected by filters. In the design of such filters, a HEMP generator is usually needed to test them.

A Marx generator is a high-voltage pulse generator first described by Erwin Otto Marx in 1924. It has many scientific uses such as HEMP protection system testing, insulation and lightning safety testing [2][3][4]. The basic operation principle of the generator is to charge capacitors in parallel and then discharge them in series. Hence a high voltage pulse can be generated. This paper describes the design of a Marx generator that can be used for HEMP filter testing. The threestage generator can produce pulses with a peak voltage of 25
$\mathrm{kV}$ and a width of $230 \mathrm{~ns}$ on a $10 \Omega$ resistor. Since the pulse width is very narrow, its frequency spectrum is very wideband. Parasitic effects of those components used in the generator cannot be ignored. The paper will describe how to establish a circuit model for each component and use the circuit models to design a Marx generator.

In this paper, the theory of generating standard compliant pulses is discussed in Section 2. The practical design and the measured results are described in Section 3. Discussions and conclusions are given in the final section.

\section{Theoretical Marx Generator Design}

The Marx generator circuit generates high voltage pulses by charging a number of capacitors in parallel first as shown in Figure 1. At the beginning the capacitors $\mathrm{C}_{\text {charge }}$ are charged by the DC power supply through charging resistors $\mathrm{R}_{\text {charge }}$. When the spark gaps are triggered, the capacitors will be discharged simultaneously in series to generate a high voltage pulse.

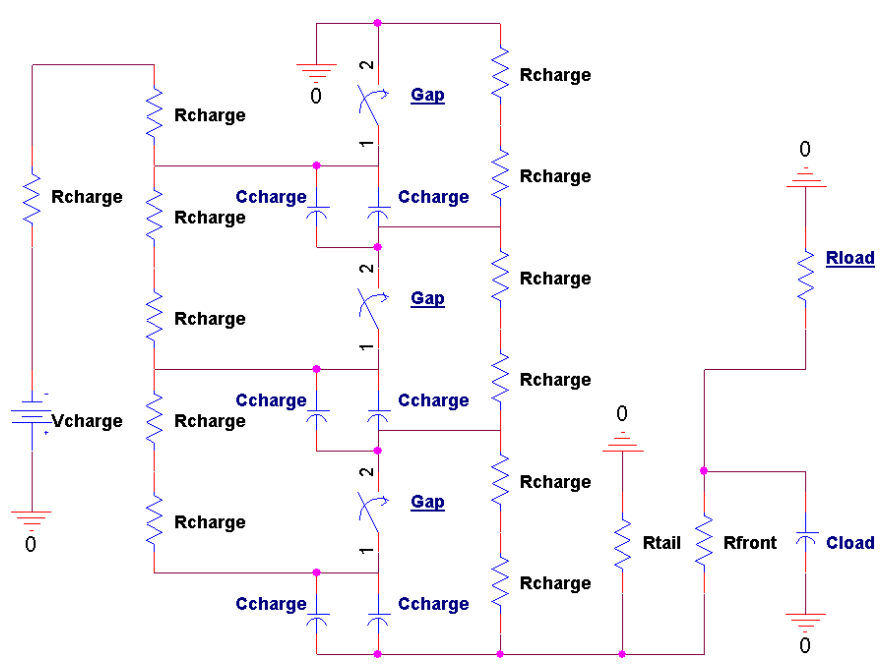

Figure 1. The circuit diagram of a typical Marx generator

To meet HEMP protection specifications, such as MIL-STD 188-125 [5], the generated pulses should have a rise time of less than $20 \mathrm{~ns}$ and a Full-Width at Half-Maximum amplitude (FWHM) of $500 \mathrm{~ns}$. The pulses should have a double 
exponential waveform as shown in Figure 2(a). While a simple RC circuit generates a single exponential waveform, the double exponential waveform can be obtained by the addition of another resistor and capacitor to the circuit as shown in Figure 2(b). The waveform can be expressed by

$$
v(t)=V\left(e^{-\alpha t}-e^{-\beta t}\right)
$$

where, on the condition that $\mathrm{R}_{2}<<\mathrm{R}_{1}$,

$$
\begin{gathered}
\alpha=\frac{1}{R_{1}\left(C_{1}+C_{2}\right)} \\
\beta=\frac{C_{1}+C_{2}}{R_{2} C_{1} C_{2}} \\
V=V_{0} \eta=V_{0} \frac{C_{1}}{C_{1}+C_{2}}
\end{gathered}
$$

Here the voltage $\mathrm{V}$ on the capacitor $\mathrm{C}_{1}$ is equivalent to the high-voltage generated by the capactors $C_{\text {charge }}$ in series as shown in Figure $1 . R_{1}$ is equivalent to $R_{\text {tail }}$ in Figure $1, R_{2}$ is equivalent to $R_{\text {front }}$ and $C_{2}$ is equivalent to $C_{\text {load }}$, resepectively. The produced double exponetial waveform is applied on the load $\mathrm{R}_{\text {load }}$.

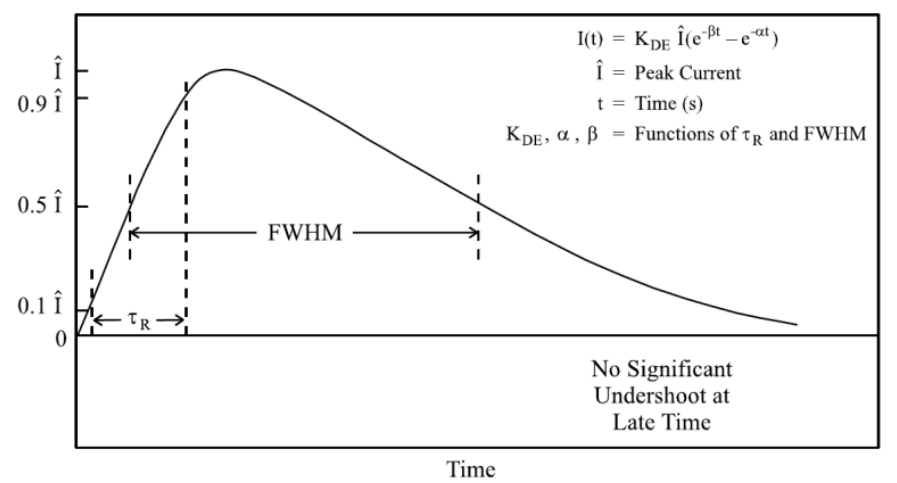

(a)

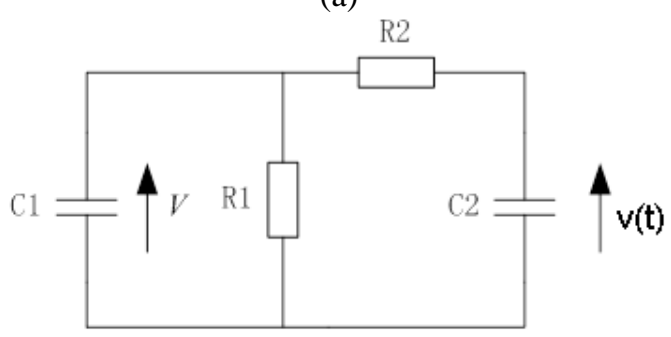

(b)

Figure 2. (a) The double exponential waveform [5] and (b) a typical circuit to generate the waveform.

Based on the circuit shown in Figure 1, a Marx generator was designed and assembled as shown in Figure 3. When the generator was first tested, the measured response, as shown in Figure 4(a), was quite different from the ideal response as shown Figure 2(a). The magnitude of the measured voltage, hence the current as well, is much higher than the theoretical value. The width was much narrower, and the pulse has high peaks of both positive and negative voltages.

The main reason is because the pulse to be generated has a narrow width, hence the spectrum is very wideband. The parasitic effect of the wires and other components significantly affect the circuit performance.

To investigate the problem, first of all, the waveform shaping components equivalent to $R_{1}, R_{2}$ and $C_{2}$ shown in Figure 2(b) was removed from the system and a $200 \mathrm{k} \Omega$ resistor was used as the load. The pulse with a relatively much wider width will be generated. The measured response is shown in Figure 4(b). It can be seen that the width of the pulse is $1.6 \mathrm{~ms}$. The curve is very smooth and agrees very well with calculation.

Therefore, to generate a narrow width pulse, the design of the generator needs to be improved by taking account of the parasitic effect. This will be discussed in the next section.

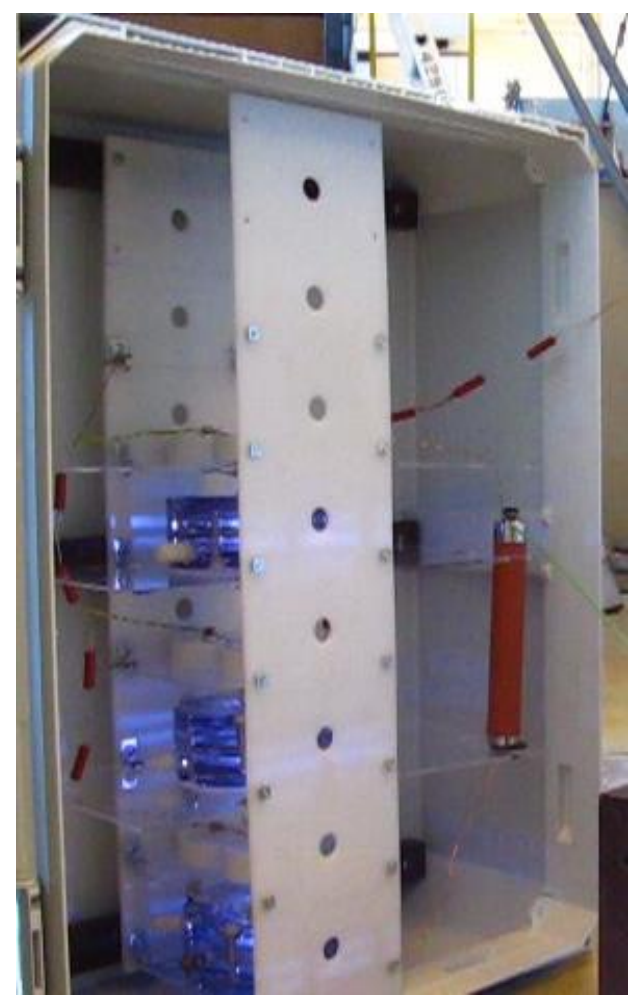

Figure 3. A photograph of the assembled generator, a transient discharge (light blue sparks) was captured.

\section{Improved Design and Experimental Results}

The response of the Marx generator should be a double exponential waveform. The rise time of the pulse is less than $100 \mathrm{~ns}$, so the bandwidth of spectrum is from DC up to tens of $\mathrm{MHz}$. The parasitic parameters of the parts and wires cannot be neglected and the circuit simulation should be improved. Figure 5(a) shows the design using improved PSpice circuit models [6][7][8][9]. The parasitic inductance of the wires, 
and both parasitic inductance and capacitance of the resistors were taken into account.

In the design, a PSpice circuit model is established for each of the components and each section of the wires. A one-stage Marx generator was constructed and measured to verify and optimize the proposed circuit models. The verified and tuned circuit model was then used to design a second-stage Marx Generator. The circuit models are further optimized and tweaked. Eventually the three-stage prototype Marx generator was designed using the verified circuit models. The simulated response of the improved design is shown in Figure 5(b). It can be seen that the generator can generate pulses with a magnitude of $23 \mathrm{kV}$ on a $10 \Omega$ resistor. The rise time is less than $100 \mathrm{~ns}$ and the FWHM is about $250 \mathrm{~ns}$.

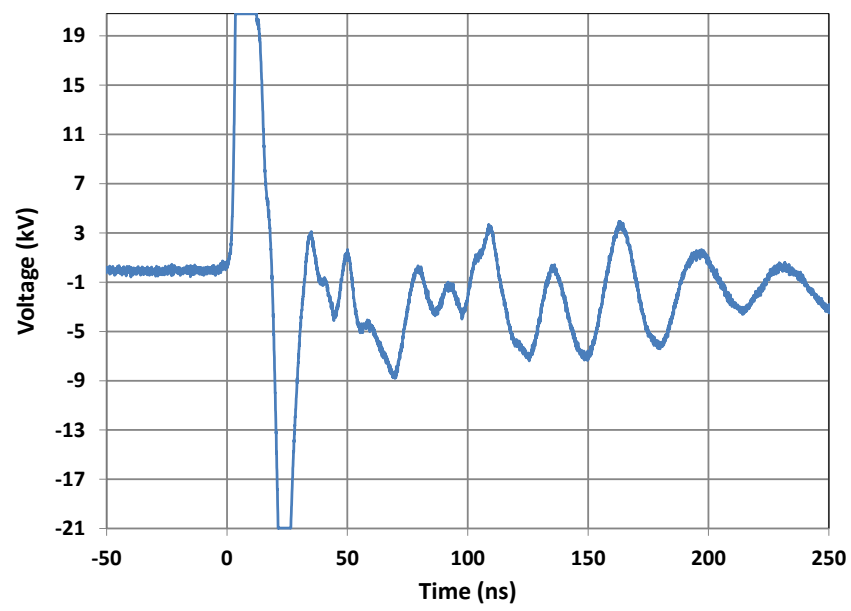

(a)

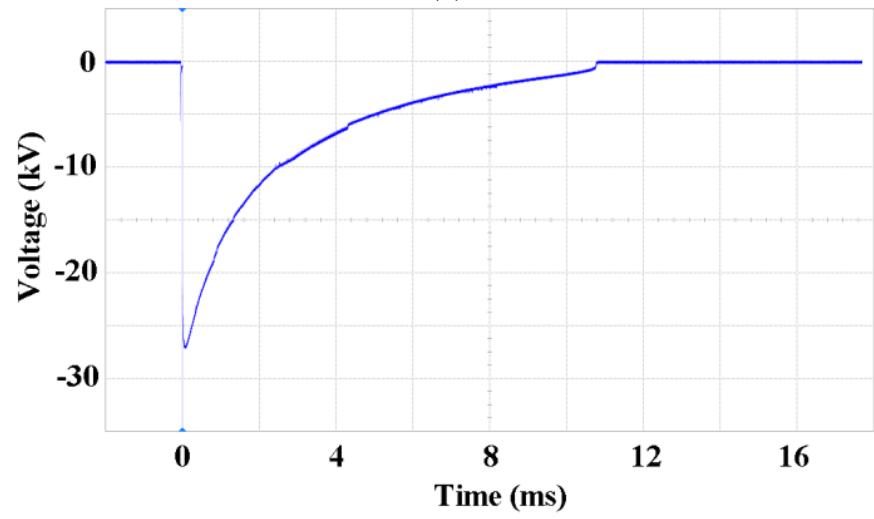

(b)

Figure 4. (a) The measured pulse generated by the generator of the initial design. (b) a wide width pulse generated by the generator when the load is a $200 \mathrm{k} \Omega$ resistor.

The measured response of the generator is shown in Figure 5(c). The pulse has an FWHM of 230 ns. The rise time is about $20 \mathrm{~ns}$. Except for the front spikes [10], the peak voltage is about $25 \mathrm{kV}$ on a $10 \Omega$ resistor, which is in very good agreement with the simulated one. It should be noted that the output voltages have negative values. If desired, positive voltages as shown in Figure 2(a) can be generated by either changing how the capacitors are connected or simply changing the polarity of the power supply.
The Marx generator was also tested with a HEMP filter. The measurement setup is shown in Figure 6(a). The HEMP filter was developed by MPE Limited. The filter was a standard four-line (three-phase) 32 A mains power line HEMP filter. All lines are individually filtered and feature inductive input to offer both good continuous wave electromagnetic compatibility (EMC) performance and superior transient handling performance on supplies with low source impedance. The filter has very low residual pulse current for high safety margin, to be verified as shown below.

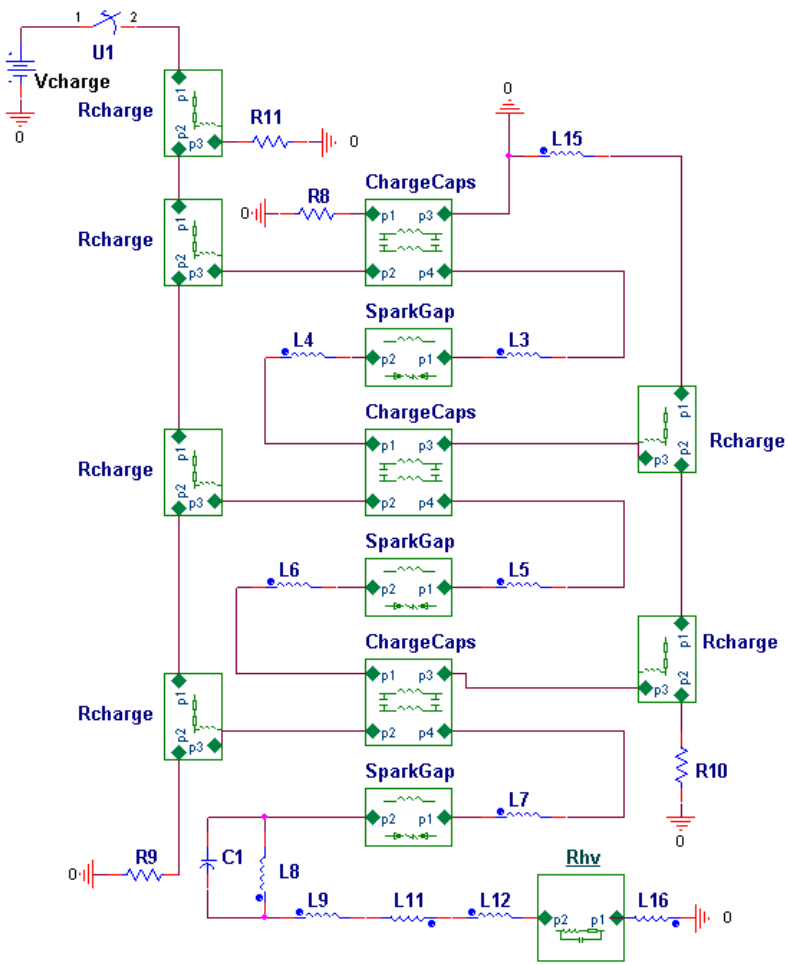

(a)

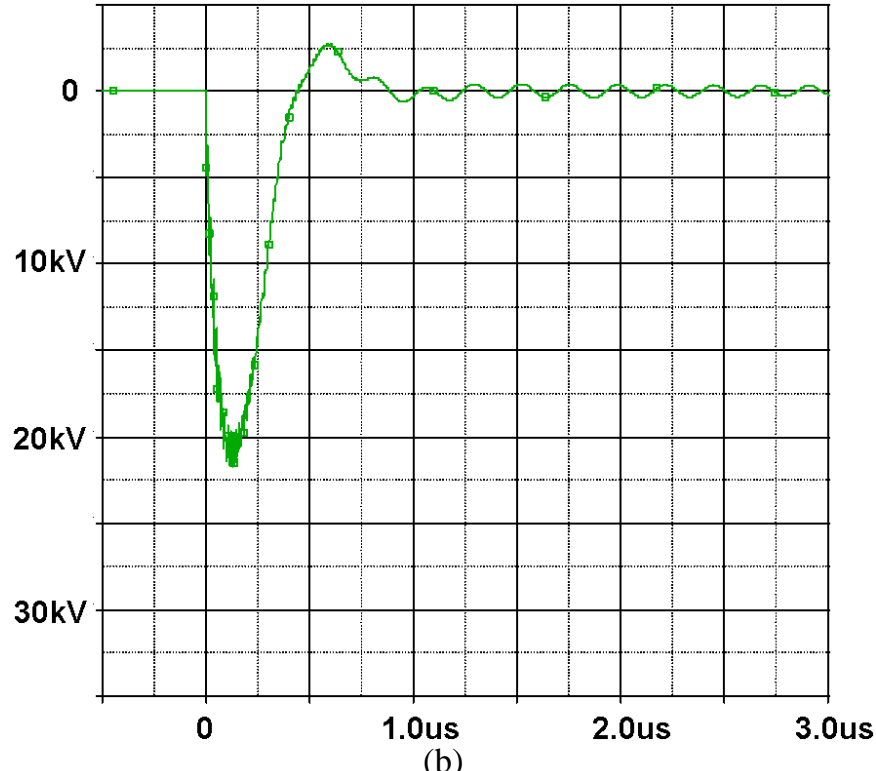

(b) 


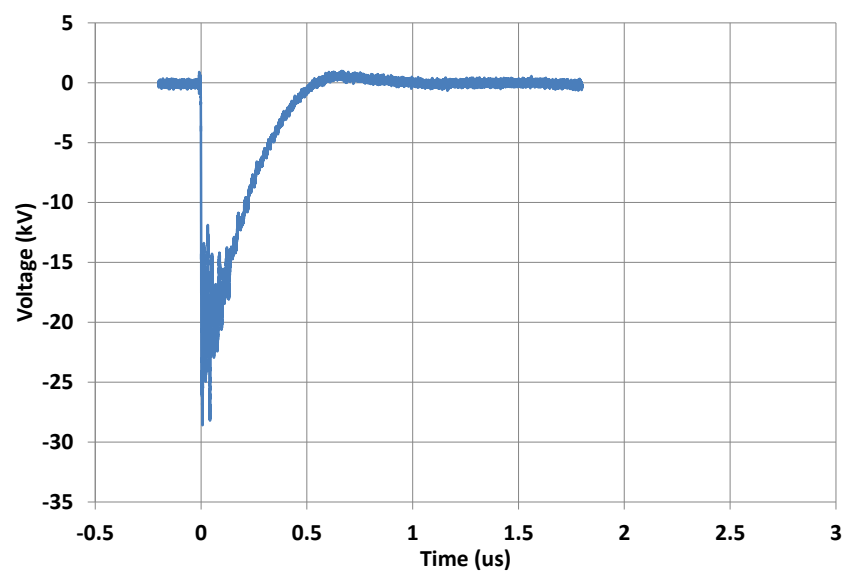

(c)

Figure 5. (a) The circuit diagram of the Marx generator using circuit models to take account of the parasitic effect of all components, (b) the simulated pulse and (c) the measured pulse generated by the generator.

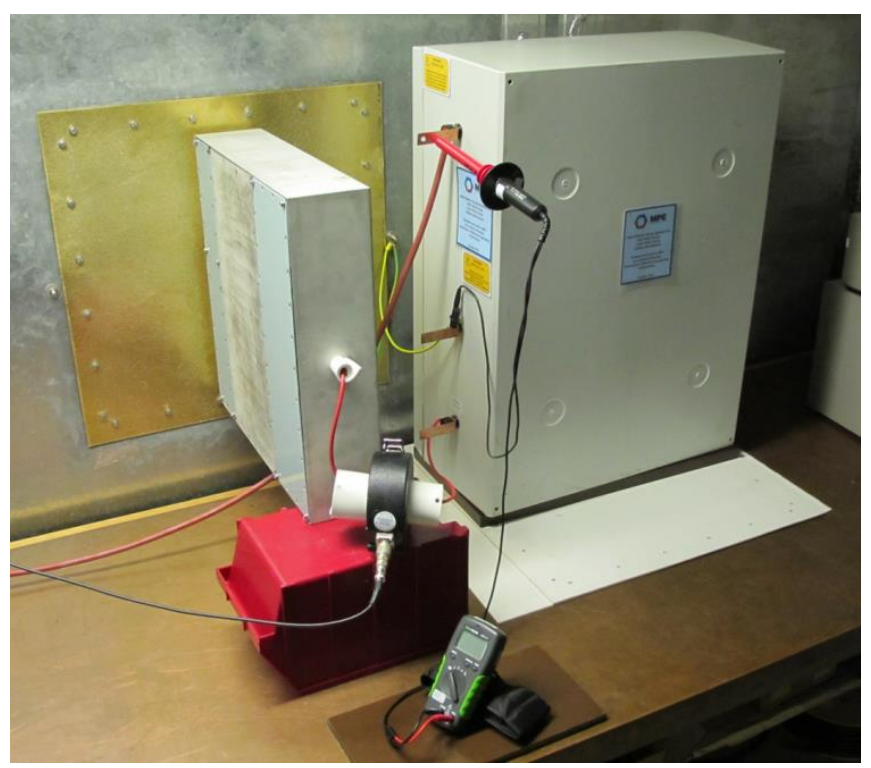

(a)

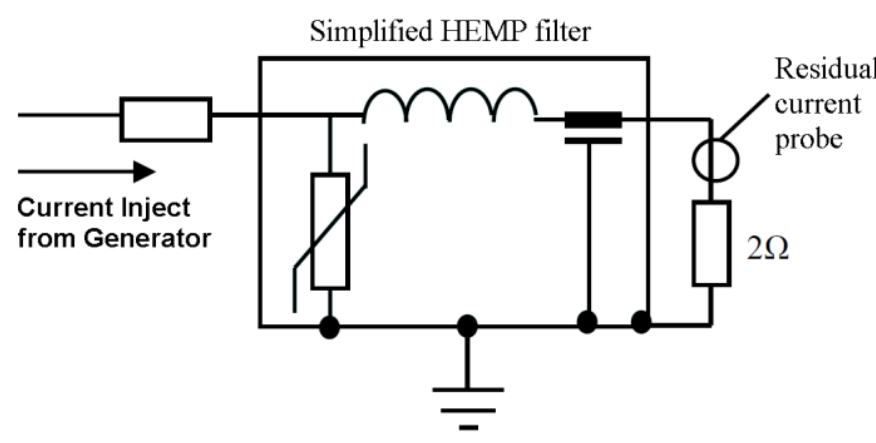

(b)

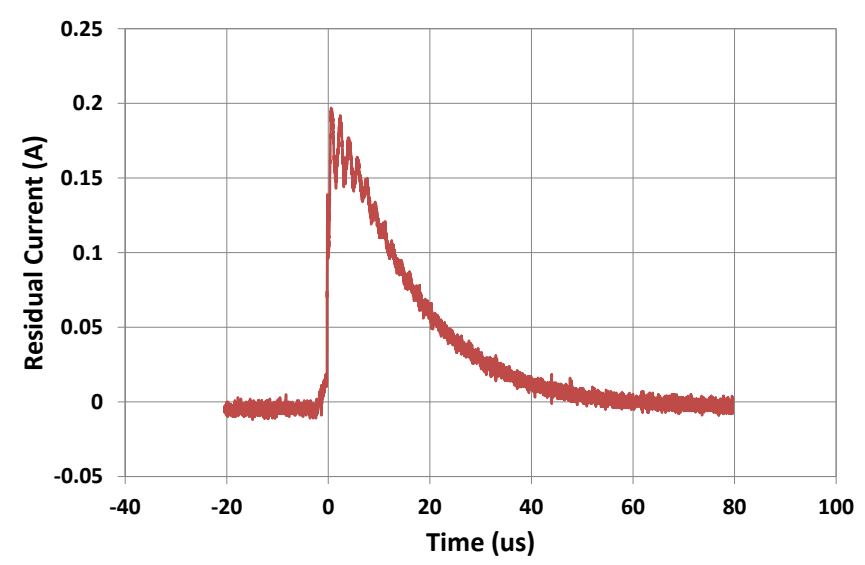

(c)

Figure 6. (a) A photograph of the measurement setup to test the generator with a HEMP filter, (b) schematic circuit diagram of the measurement setup and (c) measured residual current waveform on the $2 \Omega$ resistor.

In the test, the output of the Marx generator was connected to the input of the HEMP filter. The output of the HEMP filter was connected to a $2 \Omega$ resistor. The equivalent circuit diagram of the measurement setup is shown in Figure 6(b). The current on the $2 \Omega$ resistor was monitored using a current probe. The measured residual current is shown in Figure 6(c). It can be seen that the highest residual current is less than 0.2 A, which indicated the HEMP filter would be able to very effectively protect any HEMP with an injection of a few $\mathrm{kA}$.

\section{Conclusions}

The design of a Marx Generator is introduced in this paper. The generator can generate high altitude pulses up to $25 \mathrm{kV}$ on a $10 \Omega$ resistor. The rise time is $20 \mathrm{~ns}$ and the pulse width is about $230 \mathrm{~ns}$. Since the pulse width is very narrow and its frequency spectrum is very wideband, it was necessary to consider parasitic effect of components in the design. In the design, the circuit model of each component in the generator is proposed and verified by experiment using a one-stage generator. The verified circuit models were then used to construct the three-stage Marx generator.

The measured response of the generator was in very good agreement with the simulated one using the proposed circuit models. The developed generator was further tested with a HEMP filter. In future work, the generator can be further improved to be fully compatible with HEMP protection specifications.

\section{Acknowledgements}

The authors would like to thank the Knowledge Exchange Scheme of the University of Liverpool and MPE Limited, UK for their financial support of this project. 


\section{References}

[1] A. J. Nalborczyk, "HEMP filter design to meet MILSTD-188-125 PCI test requirements," Electromagnetic Interference \& Compatibility, INCEMIC 2008. 10th International Conference on , vol., no., pp.205-209, 2627 Nov. 2008.

[2] Hongtao Li, Hong-Je Ryoo, Jong-Soo Kim, Geun-Hie Rim, Young-Bae Kim, and Jianjun Deng, "Development of Rectangle-Pulse Marx Generator Based on PFN", IEEE Transactions on Plasma Science, VOL. 37, NO. 1, JANUARY 2009.

[3] A. A. Neuber, Y. J. Chen, J. C. Dickens, M. Kristiansen, "A Compact, Repetitive, 500kv, 500 J, Marx Generator", Pulsed Power Conference, 2005 IEEE

[4] Archana Sharma, Senthil Kumar, Sabyasachi Mitra, Vishnu Sharma, Ankur Patel, Amitava Roy, Rakhee Menon, K. V. Nagesh, and D. P. Chakravarthy, "Development and Characterization of Repetitive 1-kJ Marx-Generator-Driven Reflex Triode System for HighPower Microwave Generation", IEEE Transactions On Plasma Science, VOL. 39, NO. 5, MAY 2011.

[5] MIL-STD-188-125-1, High-Altitude Electromagnetic Pulse (Hemp) Protection For Ground-Based $C^{A} I$ Facilities Performing Critical, Time-Urgent Missions, Department of Defence, Interface Standard, USA, 17 July 1998.

[6] J. R. Mayes and C. W. Hatfield, "Development of a sequentially switched Marx generator for HPM loads," Pulsed Power Conference, 2009. PPC '09. IEEE, pp.934937, Jun. 2009.

[7] Muhammad Saufi Kamarudin, Erwan Sulaiman, Md Zarafi Ahmad, Shamsul Aizam Zulkifli and Ainul Faiza Othman, "Impulse Generator and Lightning Characteristics Simulation using Orcad PSpice Software", Proceedings of EnCon 2008, pp. 1032-1037, Dec., Malaysia, 2008

[8] Y. E. Kolyada, O. N. Bulanchuk and V. I. Fedun, "Numerical simulation of the Marx-generator behavior on nonlinear load-high-current vaccum diode," Vopr. At. Nauki Teh., Âd.-Fiz. Issled. 5 pp.27-29, 2001.

[9] R. Sheeba, M. Jayaraju, T. K. N. Shanavas, "Simulation of impulse voltage generator and impulse testing of insulator using MATLAB Simulink," Word Journal of Modelling and Simulaiton, vol. 8, no. 4, pp. 302-209, 2012.

[10] W. J. Carey and J. R. Mayes, "Marx Generator Design And Performance", Power Modulator Symposium, 2002 and 2002 High-Voltage Workshop 